\title{
Localization of moving sound
}

\author{
STEPHAN GetZMANN \\ Ruhr-Universität Bochum, Bochum, Germany \\ AND \\ JÖRG LEWALD \\ Ruhr-Universität Bochum, Bochum, Germany \\ and Leibniz Research Centre for Working Environment \& Human Factors, Dortmund, Germany
}

\begin{abstract}
The final position of a moving sound source usually appears to be displaced in the direction of motion. We tested the hypothesis that this phenomenon, termed auditory representational momentum, is already emerging during, not merely after, the period of motion. For this purpose, we investigated the localization of a moving sound at different points in time. In a dark anechoic environment, an acoustic target moved along the frontal horizontal plane. In the initial, middle, or final phase of the motion trajectory, subjects received a tactile stimulus and determined the current position of the moving target at the moment of the stimulus by performing either relative-judgment or pointing tasks. Generally, in the initial phase of the auditory motion, the position was perceived to be displaced in the direction of motion, but this forward displacement disappeared in the further course of the motion. When the motion stimulus had ceased, however, its final position was again shifted in the direction of motion. The latter result suggests that representational momentum in spatial hearing is a phenomenon specific to the final point of motion. Mental extrapolation of past trajectory information is discussed as a potential source of this perceptual displacement.
\end{abstract}

The ability to localize objects in motion is a basic function of human perception. Up to now, the localization of moving objects has mostly been studied in the visual domain, whereas in the auditory domain, research has predominantly focused on questions of motion detection and discrimination (e.g., Lutfi \& Wang, 1999; Strybel, Manligas, \& Perrott, 1992; for a review, see Gilkey \& Anderson, 1997) and of auditory motion aftereffects (e.g., Dong, Swindale, Zakarauskas, Hayward, \& Cynader, 2000; Grantham, 1992; Neelon \& Jenison, 2003). Only a few studies have investigated the localization of an acoustic object during its motion.

In an initial approach to this question, Perrott and Musicant (1977) presented a sound stimulus that rotated counterclockwise around the subject's head. Using a numerical scale, their subjects estimated the horizontal position of the sound source at the moment when the sound started (i.e., its onset position) and the moment when it ceased (i.e., its offset position). In the results, both the apparent onset and offset positions were mainly displaced in the direction of motion. The magnitude of onset displacement increased with the velocity of the sound source (between $90 \% / \mathrm{sec}$ and $600 \% \mathrm{sec}$ ), whereas the offset displacement did not follow a simple rule: In general, offset positions were less displaced than onset positions with shorter sound durations (50 and $100 \mathrm{msec}$ ), but more displaced with longer sound durations (150 and $300 \mathrm{msec}$ ).
In two recent studies, systematic mislocalizations of the onset and offset positions of moving sounds were found also with much lower target velocities than those used by Perrott and Musicant (1977). In both of these recent studies, subjects were presented with an acoustic target that moved from left to right or from right to left along the frontal horizontal plane, covering a distance of $36^{\circ}$ or $40^{\circ}$, in a completely dark and anechoic environment. In Getzmann, Lewald, and Guski (2004), subjects pointed to the final position of targets moving at a constant velocity of $8 \%$ sec or $16^{\circ} / \mathrm{sec}$. In Getzmann (2005b), relative judgments were used to determine the perceived onset position of targets moving at $12 \% \mathrm{sec}$. As in Perrott and Musicant's study, both onset and offset positions were displaced in the direction of motion. These displacements have been related to analogous effects in the visual modality: the Fröhlich effect and representational momentum.

The Fröhlich effect (Fröhlich, 1923) is a systematic mislocalization of the onset position of a moving visual object, typically a displacement in the direction of motion (see, e.g., Müsseler \& Aschersleben, 1998; Müsseler, Stork, \& Kerzel, 2002). Several explanations have been proposed that may account for this effect. Attention-based approaches assume, for example, that the initial position of motion receives less attention. Given that attention is a necessary prerequisite for conscious processing of spatial information, the first part of the trajectory is missed, and the perceived

S. Getzmann, stephan.getzmann@ruhr-uni-bochum.de 
onset position appears to be shifted in the direction of motion (Müsseler \& Aschersleben, 1998). In a similar way, an auditory spatial attention mechanism could explain the Fröhlich effect in spatial hearing (Getzmann, 2005b).

The representational momentum becomes evident when observers are asked to determine the final position of an object presented in motion (Freyd \& Finke, 1984; for reviews, see Hubbard, 2005; Thornton \& Hubbard, 2002). Typically, observers misjudge the position as having been farther along the implied motion trajectory. This effect has been termed representational momentum because of an analogy between physical momentum and a dynamic aspect of the mental representation of target position (Freyd, 1987). The source of this forward displacement is still under debate: Numerous studies on visual representational momentum have revealed that displacements are not solely based on principles of internalized dynamics (i.e., the idea that the mental extrapolation of motion is analogous to the physical momentum of real-world objects), but instead reflect a wide range of influences from characteristics of the target, display, context, and observer (e.g., Hubbard, 2006; Kerzel, 2005). Recent findings have demonstrated that eye movements do not contribute to the displacement effect in spatial hearing (Getzmann, 2005a; but see Kerzel, 2006). Instead, it was hypothesized that the auditory motion initiates a neural process that results in a shift of the representation of auditory space in the direction of motion. This approach is supported by neurophysiological findings in animal studies providing evidence that sound motion can induce shifts of receptive fields in spatially selective auditory neurons (Malone, Scott, \& Semple, 2002; Wilson \& O’Neill, 1998).

To clarify the nature of representational momentum in spatial hearing, it is relevant to know when the induced shift in the representation of auditory location arises. On the one hand, the displacement does not manifest itself before the end of motion. The localization of a moving target may be precise as long as the target exists, whereas displacements of the final position may occur after the target has disappeared. On the other hand, the shift could already be emerging during motion. That is, the spatial representation of the moving target could be distorted, even though the target is still present. If so, the displacement could increase during the course of motion or, alternatively, a constant forward displacement could exist from the beginning until the end of motion.

In this context, a study of Joordens, Spalek, Razmy, and van Duijn (2004) on visual representational momentum might be of interest: In a clock-watching task, observers were presented with a dot moving in clockwise direction. During the motion, the background color of the display changed. After disappearance of the target, the observers localized the position where the target dot was when the display changed color. Interestingly, a slight, nonsignificant backward displacement of the target position was found, but no forward displacement (Experiment 1). However, a significant forward displacement occurred when observers localized the final target position (Experiment 3 ). Thus, one may conclude that forward displacements associated with representational momentum occur at the end of motion - that is, after the target has ceased. Alternatively, as proposed by Joordens et al., goaloriented controlled processes may be active during motion that partly compensate for forward displacements.

In a study on localization of moving sound, Mateeff and Hohnsbein (1988) found a divergent pattern of displacement: Subjects localized a sound source that moved at $15.2 \mathrm{~cm} / \mathrm{sec}$ (about $15^{\circ} / \mathrm{sec}$ ) over a horizontal path $60 \mathrm{~cm}$ in length. The source was positioned at a distance of $57 \mathrm{~cm}$ in front of the subject. Subjects localized the acoustic target at different points in time with reference to stationary visual stimuli. In general, the target was mislocalized in the direction of motion, and most importantly, this forward displacement increased in the course of motion: At the starting point of motion, there was a slight shift of the perceived target position in the direction opposite to motion, whereas at the final point there was a displacement in the direction of motion of up to $10^{\circ}$. Thus, it seems that the forward displacement of the final position observed after the target's disappearance already exists during motion and increases with the motion duration.

However, the method employed by Mateeff and Hohnsbein (1988) involved visual factors in addition to auditory ones, in that subjects judged the current position of the target relative to a visual reference that was visible during the sound presentation. An increase in the luminance of the visual reference indicated the point in time when the target position had to be judged. Thus, it is possible that auditory-visual crossmodal interactions, in particular a visual bias of the sound localization (the so-called ventriloquism effect), were superimposed over potential genuine auditory effects (see, e.g., Lewald, Ehrenstein, \& Guski, 2001; Lewald \& Guski, 2003; Shelton \& Searle, 1980; Warren, 1970). Another critical factor in Mateeff and Hohnsbein's study is the potential influence of different perceptual latencies of the auditory event (the moving acoustic target) and the visual event (the increase in luminance of the visual reference). It is well known that the neural processing of visual information is delayed in comparison with that of auditory information (see, e.g., King \& Palmer, 1985). Thus, the observed mislocalization could be, at least in part, due to this visual delay.

In the present study, two different psychophysical methods were employed to investigate the influence of motion on the perceived position of an acoustic target. Subjects heard a sound that moved at a moderate velocity $(12 \% \mathrm{sec})$ either from left to right or from right to left along the frontal horizontal plane. During motion, the subjects received a short tactile stimulus that, unlike the visual stimulus used by Mateeff and Hohnsbein (1988), did not provide any relevant spatial information. In Experiment 1A, relative judgments were made with respect to the perceived position of the target at the moment of tactile stimulation; subjects were asked to compare the current target position with a stationary visual reference provided before and during the auditory motion. In Experiment 1B, a pointing method was employed in absolute darkness. As was done in our previous studies (Getzmann, 2005a; Getzmann et al., 2004), subjects directed a hand pointer toward the target position at the moment of tactile stimulation. 


\section{EXPERIMENT 1}

\section{Method}

Subjects. Twelve subjects participated in the experiment. All of them ( 6 female, 6 male; age range $20-30$ years, mean 24.4 years) had normal hearing and normal or corrected-to-normal vision. They were unaware of the scientific background of the study. One session (lasting $2 \mathrm{~h}$, including rest breaks) was conducted with each subject. Prior to their inclusion in the study, all subjects gave informed consent. The experiments reported in this study were noninvasive and were performed in accordance with the ethical standards laid down in the 1964 Declaration of Helsinki.

Apparatus and Stimuli. The subject sat on a chair in an absolutely dark, soundproof, and anechoic room $(4.4 \mathrm{~m}$ wide $\times 5.4 \mathrm{~m}$ long $\times 2.1 \mathrm{~m}$ high), which was insulated by $40 \mathrm{~cm}$ (height) $\times 40 \mathrm{~cm}$ (depth) $\times 15 \mathrm{~cm}$ (width at base) fiberglass wedges on each of the six sides. A suspended mat of steel wires served as the floor. The ambient background noise level was below $20 \mathrm{~dB}(\mathrm{~A})$ sound pressure level. The position of each subject's head was stabilized by a custom-made chin and forehead rest. In front of the subject, at a constant distance of $1.5 \mathrm{~m}$ from the center of the head, 91 broadband loudspeakers (Visaton SC 5.9, $5 \times 9 \mathrm{~cm}$ ) were mounted in the subject's horizontal plane. The azimuth of the loudspeakers ranged from $-90^{\circ}$ (left) to $+90^{\circ}$ (right) in steps of $2^{\circ}$, with the center loudspeaker at $0^{\circ}$. All loudspeakers were selected on the basis of comparable efficiency and frequency response curves.

The target sound was generated digitally and converted to ana$\log$ form via a PC-controlled, 16-bit soundcard (Creative Sound Blaster 16) at a sampling rate of $44.1 \mathrm{kHz}$. Sound pressure level was $66 \mathrm{~dB}(\mathrm{~A})$, as measured at the subject's head position using a Brüel and Kjær sound level meter (Type 2226). The target sound consisted of 50-msec noise bursts (band-pass-filtered noise; lower and upper cutoff frequencies of 1 and $3 \mathrm{kHz}$, respectively). Apparent auditory motion was generated by successively activating one loudspeaker after the other along the horizontal loudspeaker arrangement (see the Procedure section below). Three white light-emitting diodes (LEDs; $7 \mathrm{mcd}, 3 \mathrm{~mm}$ ) served as visual reference stimuli in Experiment 1A. The LEDs, which did not illuminate the surrounding loudspeakers, were attached below the loudspeakers at $-20^{\circ}, 0^{\circ}$, and $20^{\circ}$ relative to straight ahead. In Experiment 1B, a hand pointer was mounted in front of the subject. This swivel pointer consisted of a metal rod that the subject could rotate in the horizontal plane. A key was mounted on the upper side of the rod. The azimuthal angle of the pointer was recorded by a potentiometer as soon as the key was pressed (for further details, see Lewald, Dörrscheidt, \& Ehrenstein, 2000).

Tactile stimulation was produced electromagnetically by a custom-made pressure transducer. This device consisted of a small box ( $3 \mathrm{~cm}$ wide $\times 7 \mathrm{~cm}$ long $\times 4 \mathrm{~cm}$ high) that was attached to a subject's left or right forearm by means of an elastic belt. Within the box, a small electromagnet operated an outward pin (diameter $1 \mathrm{~mm}$ ) that, when activated, slightly hit the subject's arm (amplitude $1 \mathrm{~mm}$, duration $5 \mathrm{msec}$ ). The mechanism was acoustically insulated to avoid any operation noise that could be used as additional auditory information. In order to compensate for a potential localization bias that might be induced by lateral tactile stimulation, half of the subjects received the tactile stimulus on the left arm and half on the right arm. The timing of the stimuli and the recording of the subject's responses were controlled by custom-written software.

Procedure in Experiment 1A: Relative judgments. Each session consisted of three main blocks. In the first block, subjects were asked to determine the position of the moving acoustic target with reference to the visual stimulus at the moment of tactile stimulation (Figure 1A). Each trial started with the onset of a single visual reference stimulus (illuminated LED, duration $7.8 \mathrm{sec}$ ). The positions of the visual stimulus $\left(-20^{\circ}, 0^{\circ}\right.$, or $\left.20^{\circ}\right)$ did not vary randomly, but were presented block by block, with the order of presentation counterbalanced between subjects. One second after the onset of the LED, the target sound started and immediately began to move at a constant velocity $(12 \% \mathrm{sec})$ from left to right (LR) or from right to
A
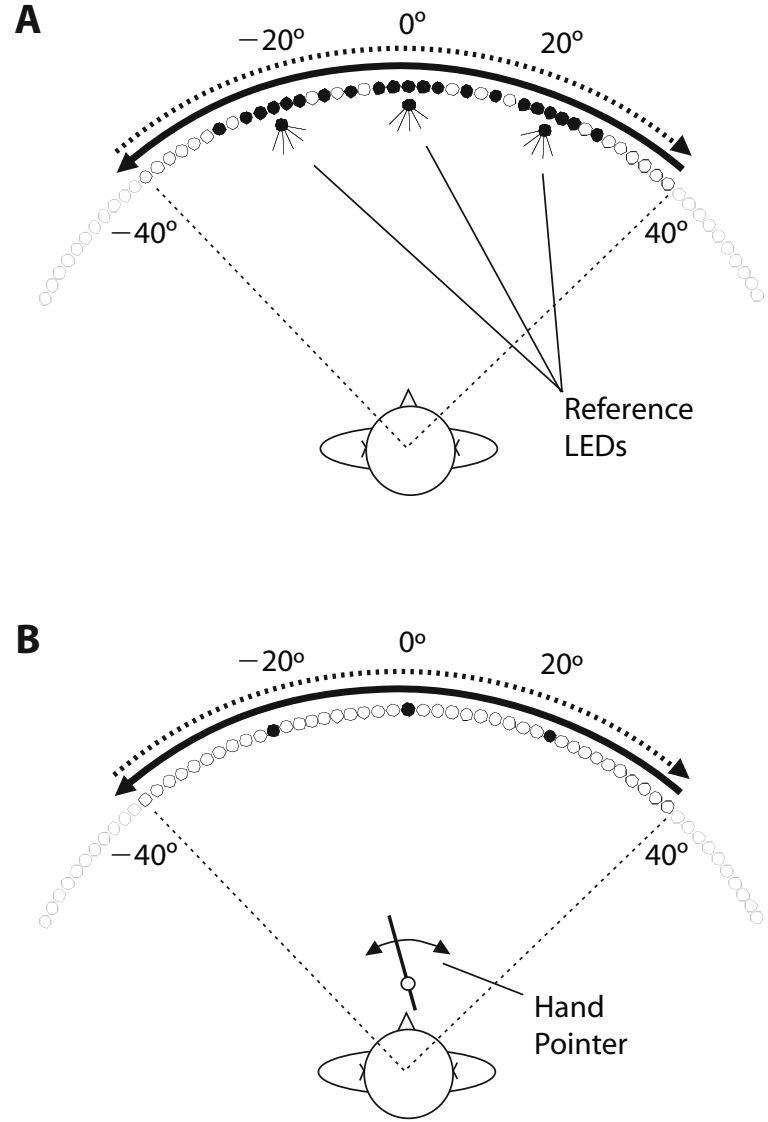

Figure 1. Schematic illustration of the trajectories of left-toright (dotted arrows) and right-to-left (solid arrows) motion. Positive positions are to the right and negative positions to the left of the subject's median plane. (A) In Experiment 1A, subjects judged the current position of the moving acoustic target at the moment they received a tactile stimulus with reference to a visual stimulus presented at $-2^{\circ}, 0^{\circ}$, or $2^{\circ}$ (reference LEDs). Actual target positions at the moment of tactile stimulation are marked (filled circles). (B) In Experiment 1B, subjects pointed at the current position of the target at the moment of tactile stimulation by using a hand pointer. Actual target positions are marked (filled circles).

left (RL), covering a distance of $80^{\circ} .{ }^{1}$ The auditory onset position was $-40^{\circ}$ with LR motion and $40^{\circ}$ with RL motion. While the auditory target moved, a short tactile stimulus (see above) was presented. Using a two-alternative forced choice paradigm, the subjects had to indicate whether the position of the auditory target at the moment of tactile stimulation was to the left or the right of the visual reference: They had to press the left key of a response box when the sound was to the left of the visual stimulus, and the right key when the sound was to the right of the visual stimulus. The tactile stimulation was exactly timed so that the distance between the current position of the auditory target and the visual reference was either $\pm 8^{\circ}, \pm 4^{\circ}$, $\pm 2^{\circ}$, or $0^{\circ}$. For example, when the visual stimulus was located at $-20^{\circ}$, the current position of the auditory target at the moment of tactile stimulation was $-28^{\circ},-24^{\circ},-22^{\circ},-20^{\circ},-18^{\circ},-16^{\circ}$, or $-12^{\circ}$ (see the filled circles in Figure 1A). At the end of the auditory motion (duration $6.8 \mathrm{sec}$ ), the visual stimulus and the sound ended simultaneously; $2.2 \mathrm{sec}$ later, the next trial started. Six LR- and six RL-motion stimuli per condition were presented in quasirandom order, resulting in a total of 252 trials $(2$ motion directions $\times 3$ visual reference positions $\times 7$ target sound positions $\times 6$ replications).

In the second block of the experiment, potential differences in the perceptual latencies of the auditory and the tactile stimulation 
were measured by employing temporal order judgments. A single, 50 -msec noise burst was presented straight ahead of subjects. In addition, a 5-msec tactile stimulus was presented either shortly before or after the noise burst. The stimulus onset asynchrony (SOA) was $\pm 120, \pm 90, \pm 60, \pm 30$ or $0 \mathrm{msec}$. Each SOA was randomly presented seven times. The duration of each trial was $2.2 \mathrm{sec}$. In a two-alternative forced choice task, subjects indicated whether the acoustic stimulus started before or after the tactile stimulus.

In the third block, stationary acoustic targets were presented, in order to control for a potential response bias in sound localization. As in the first block, each trial started with the onset of a single visual reference, located at $-20^{\circ}, 0^{\circ}$, or $20^{\circ}$. One second after the start of the visual reference (duration $4 \mathrm{sec}$ ), a stationary acoustic target (3-sec noise burst) was presented at one of the positions where the moving acoustic target in the first block was located at the moment of tactile stimulation. For example, with the visual reference located at $-20^{\circ}$, the stationary acoustic target was presented at $-28^{\circ},-24^{\circ},-22^{\circ},-20^{\circ}$, $-18^{\circ},-16^{\circ}$, or $-12^{\circ}$. By pressing one of the response keys, subjects indicated whether the target sound was to the left or the right of the visual reference. One second after the end of the visual reference, the next trial started. The third block consisted of a total of 126 trials ( 3 visual reference positions $\times 7$ target sound positions $\times 6$ replications).

No feedback was given at any time during the experiment. Trials in which the subject failed to respond were repeated at the end of the block.

Procedure in Experiment 1B: Pointing. Experiment 1B was conducted with the same subjects on a different day within 1 week after Experiment 1A. The procedure was as in Experiment 1A, except that the position of the moving acoustic target was localized by a manual pointing operation (Figure 1B). Each trial started with the onset of the target sound, which immediately began to move in an LR or RL direction. During the motion (duration $6.8 \mathrm{sec}$ ), a tactile stimulus was presented as in Experiment 1A. The location of the auditory target at the moment of tactile stimulation was $-20^{\circ}, 0^{\circ}$, or $20^{\circ}$. Immediately after the tactile stimulation, the subject directed the hand pointer as accurately as possible toward the target position. The next trial started $3.2 \mathrm{sec}$ after motion offset, and each trial lasted for $10 \mathrm{sec}$. As in Experiment 1A, additional "stationary" trials were presented at the end of the session, in which the subjects pointed to target sounds (duration $6.8 \mathrm{sec}$ ) that were statically presented at $-20^{\circ}, 0^{\circ}$, or $20^{\circ}$. Six stimuli per condition were presented in quasirandom order, resulting in a total of 54 trials ( 3 target positions $\times 3$ motion conditions [LR, RL, stationary] $\times 6$ replications).

\section{Results}

Experiment 1A: Relative judgments. For calculating shifts in perceived target positions, a nonlinear regression analysis was used (Cox, 1970). The percentage of "right" judgments was determined as a function of the angle between the acoustic target position and the visual reference stimulus. The percentages from each subject were then fitted to the sigmoid equation $f=100 /[1+$ $e^{-k\left(x-x_{0}\right)}$, where $f$ is the frequency of "right" judgments, $x$ is the angle of the target position relative to the visual reference, $k$ is the slope of the psychometric function, and $x_{0}$ is the bias coefficient (i.e., the angle at which $f$ is $50 \%$ ). As illustrated in Figure 2, the fitted curves appear sigmoidal in linear plots of percentage of "right" judgments versus the relative angle between target position and visual reference. This figure shows the percentage of "right" judgments and the fitted psychometric functions for the central position of the visual reference. As can be seen, the psychometric function obtained with RL-motion targets was shifted to the right, whereas those obtained with LR motion and - to a lesser degree - with stationary targets were shifted to the left.

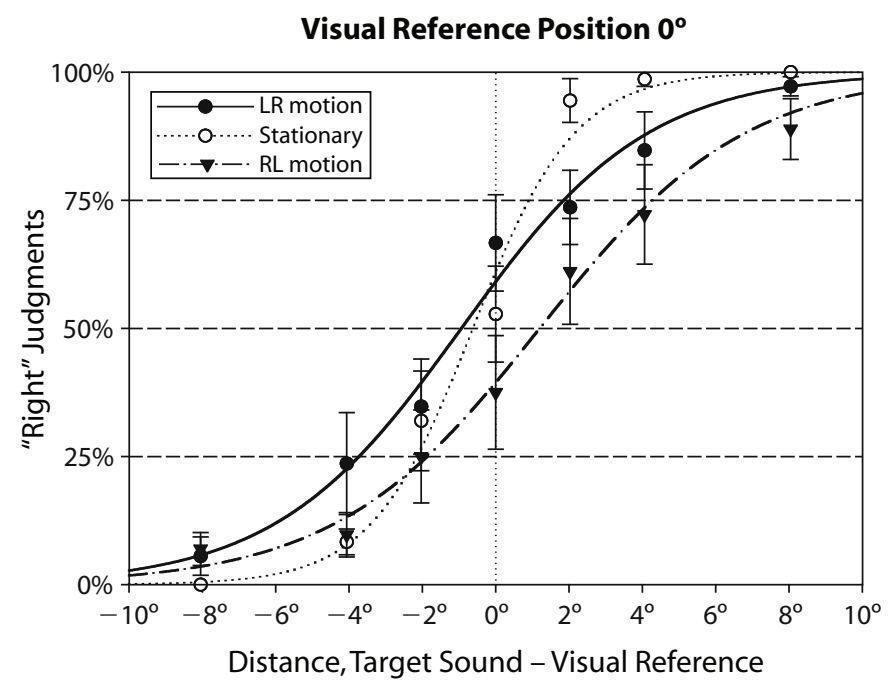

Figure 2. Percentage of "right" judgments in Experiment $1 \mathrm{~A}$ as a function of the angle of the acoustic target position relative to the visual reference stimulus, shown exemplarily for the central reference position. Positive angles indicate that the current target position (i.e., at the moment of tactile stimulation) was on the right of the visual reference stimulus, and vice versa for negative angles. Each data point indicates the percentage of decisions in which the target was perceived to the right of the reference. The acoustic target moved in either the left-to-right (LR) or right-to-left (RL) direction; alternatively, the target was presented statically. The data represent the mean values $( \pm S E)$ of 12 subjects. Sigmoids illustrate the fitted curves for each condition. 
The bias coefficient $x_{0}$ resulting from the fit is an indicator of shifts of the psychometric function, thus providing a measure of perceptual displacement: An $x_{0}$ of 0 indicates veridical judgments; if $x_{0}$ is positive (or negative) - that is, if the psychometric function is shifted to the right (or left) - subjects more frequently judged the target position to be to the left (right) of the visual reference. From the bias coefficient of the psychometric function, the shift of the perceived target position was calculated for each subject and each motion condition. In order to cancel any bias in stationary sound localization, displacements were corrected by subtracting the shifts measured with stationary targets from those measured with moving targets. Finally, forward displacements were calculated by taking the corrected displacements as positive when the target position was shifted in the direction of motion, and negative when shifted in the direction opposite motion.

Differences in perceptual latencies were calculated in a corresponding way: The percentage of "acoustic stimulus before tactile stimulus" judgments was determined as a function of the SOA of the acoustic and tactile stimuli and was fitted to a sigmoid equation. The resulting bias coefficient $x_{0}$ was defined as a measure of the difference in perceptual latencies. This latency value was taken as positive when the acoustic stimulus was perceived before the tactile one, and vice versa. A positive latency value would thus cause a constant shift of the perceived position of the moving acoustic target in the direction of motion: That is, although actually presented at the same position, the acoustic target was judged to have already passed the visual reference stimulus when the tactile stimulation was perceived.

The analysis of these temporal order judgments revealed only a slight difference in audio-tactile latencies (mean $+1.9 \mathrm{msec}, S E \pm 7.6 \mathrm{msec}$ ). The mean angular shift computed from the crossmodal latency difference (i.e., target velocity $\left[12^{\circ} / \mathrm{sec}\right] \times$ latency value $[1.9 \mathrm{msec}]$ ) was $0.02^{\circ}$ $\left(S E \pm 0.09^{\circ}\right)$. Similarly, the angular shift for each subject was computed by multiplying the individual latency difference by the target velocity. Individual values were then used to correct the forward displacement for each subject by subtracting the angular shift due to the latency difference from the forward displacements.

Displacements obtained with relative judgments are shown in Figure 3A. With LR motion, there was an initial backward displacement (i.e., opposite to motion) when the target passed the left reference position $\left(-20^{\circ}\right)$. This turned into a forward displacement in the final phase of motion, when the target passed the right reference position $\left(20^{\circ}\right)$. With RL motion, a forward displacement occurred in the middle phase of motion (when the target passed the central reference position, $0^{\circ}$ ) and increased in the final phase of motion. As confirmed by $t$ tests, only (forward) displacements in the final phase of motion statistically differed significantly from zero [LR motion and right reference, $t(11)=2.46, p<.05 ;$ RL motion and left reference, $t(11)=5.70, p<.001]$, whereas displacements in the middle [LR motion and central reference, $t(11)=0.24$, $p>.05$; RL motion and central reference, $t(11)=1.91$, $p>.05]$ and in the first phases of motion [LR motion and
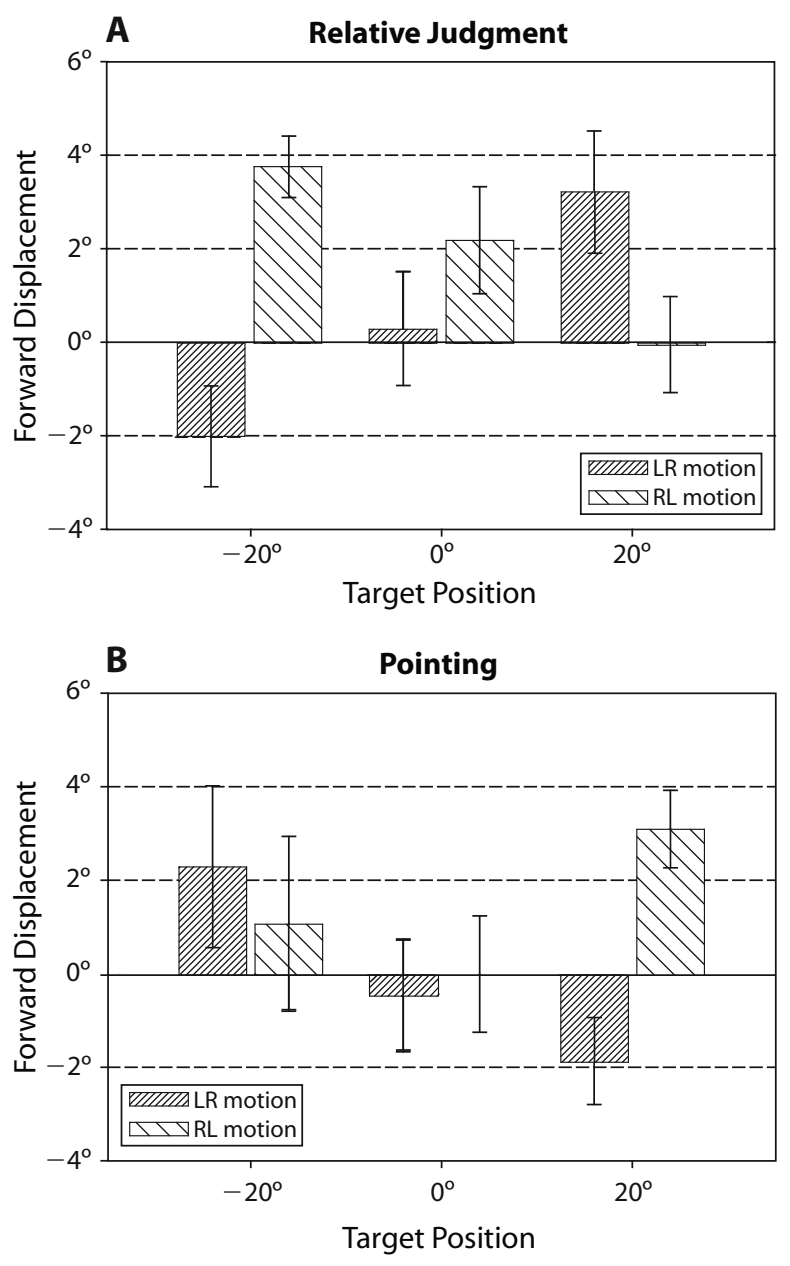

Figure 3. Mean localization displacements in Experiment 1 as a function of the target position relative to the median plane and the direction of motion, shown separately for relative judgments (A) and pointing (B). Displacements were calculated by subtracting differences obtained in the stationary conditions from those in the motion conditions. Positive angles represent displacements in the same direction as the motion (forward displacements). Error bars are $S E$ s across subjects $(N=12)$. $L R$, left to right; $R L$, right to left.

left reference, $t(11)=1.85, p>.05 ; \mathrm{RL}$ motion and right reference, $t(11)=0.05, p>.05$ ] remained nonsignificant. Repeated measures ANOVAs revealed main effects of position on displacement with both $\operatorname{LR}[F(2,22)=12.62$, $p<.01]$ and $\operatorname{RL}[F(2,22)=5.10, p<.05]$ motions.

Figure 4A shows the mean forward displacements, averaged across LR and RL motions. The plot indicates a slight backward displacement at the moment when the target has covered the first $20^{\circ}$ of the motion trajectory. After $40^{\circ}$ of motion (i.e., in the median plane), this pattern turned into a slight forward displacement, which further increased after $60^{\circ}$ of motion. However, only the final displacement differed significantly from zero $\left[3.5^{\circ}\right.$, $\left.S E \pm 0.8^{\circ} ; t(11)=4.54, p<.05\right]$, whereas the middle $\left[1.2^{\circ}, S E \pm 1.0^{\circ} ; t(11)=1.22, p>.05\right]$ and initial $\left[-1.0^{\circ}\right.$, $\left.S E \pm 0.7^{\circ} ; t(11)=1.42, p>.05\right]$ displacements did not. An ANOVA indicated a main effect of phase of motion 

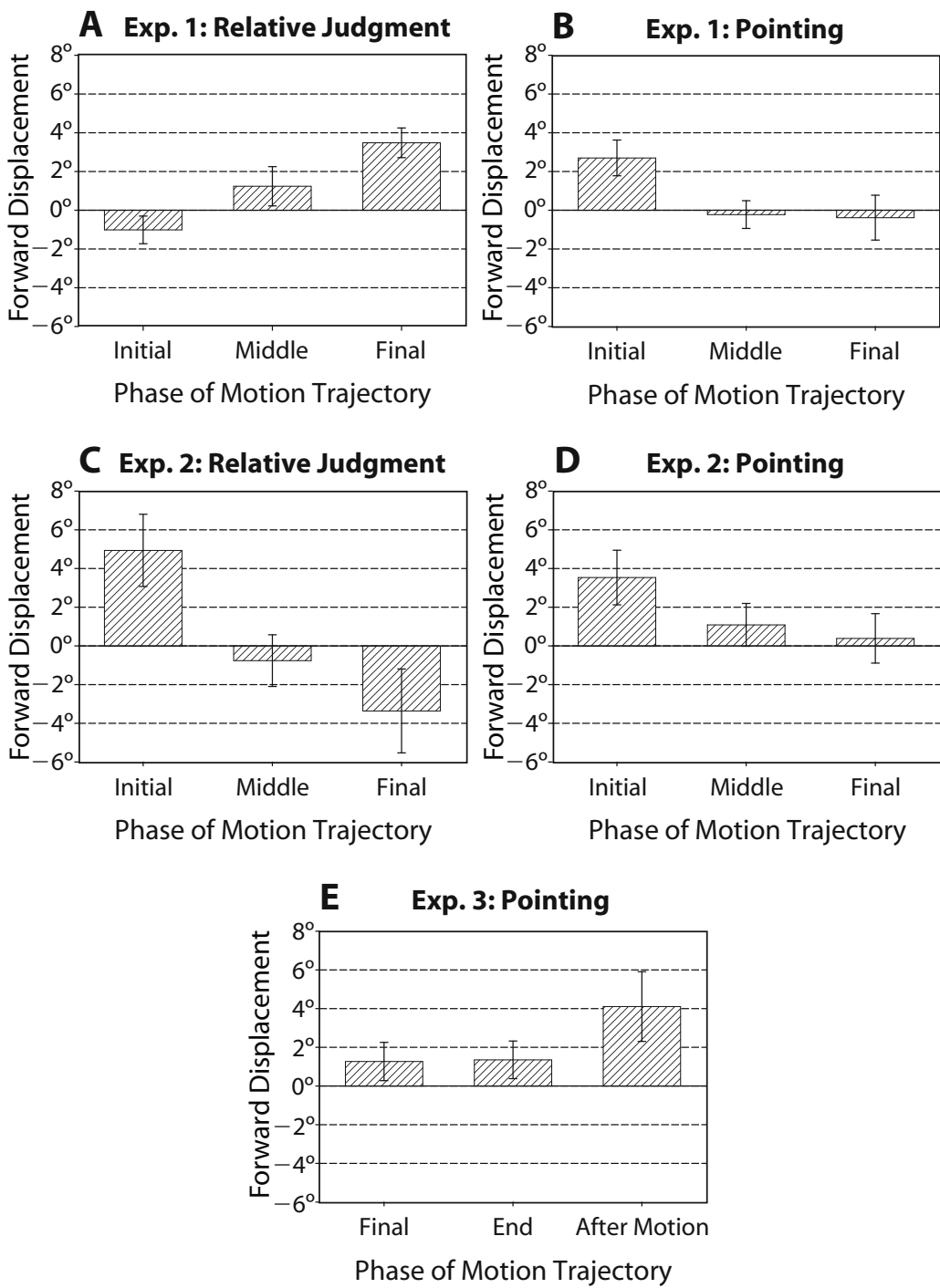

Figure 4. Forward displacements of the target position as a function of the phase of motion trajectory, shown separately for Experiments 1 (A, B), 2 (C, D), and 3 (E). The initial phase of the motion trajectory refers to the moment when the target has passed the first $20^{\circ}$ of the motion trajectory; the middle phase refers to the center of the motion trajectory; and the final phase refers to the moment when the target was $20^{\circ}$ before its final position (see Figure 1). The data are averaged across motion directions, and error bars are $S E$ s across subjects (for panels $\mathrm{A}$ and $\mathrm{B}, N=12$; for panels $\mathrm{C}, \mathrm{D}$, and $\mathrm{E}, N=11)$.

$[F(2,22)=14.51, p<.001]$. Also, post hoc $t$ tests showed that forward displacements at the initial, middle, and final phases of motion differed significantly $(p<.05)$.

Experiment 1B: Pointing. Differences between the pointing azimuth and the actual target position at the moment of tactile stimulation were measured for each target position. Also, differences between the actual position of the stationary targets and the pointing azimuth were measured. To compensate for a potential response bias in sound localization, differences with stationary targets were subtracted from those with moving targets (separated for LR and RL motions). Positive displacements were in the direction of motion and negative ones opposite the direction of motion. Furthermore, effects of audio-tactile latency differences were compensated for by subtracting shifts due to different perceptual latencies from the forward displacements, as described for Experiment 1A (see above).

Displacements observed with the pointing operation are shown in Figure 3B. With LR motion, there were nonsignificant tendencies for a forward displacement at the left reference position and a backward displacement at the right and central reference positions [difference from zero: left, $t(11)=1.34$; central, $t(11)=0.38$; right, $t(11)=1.96$; all $p \mathrm{~s}>.05]$. However, a significant effect of position, revealed by an ANOVA $[F(2,22)=3.79, p<.05]$, suggested an increase in backward displacement in the course of the motion. With RL motion, a significant forward displacement was observed at the right reference position $[t(11)=$ 
$3.78, p<.05]$, whereas displacements at the central and left reference positions did not differ from zero $[t(11)=$ $0.01 ; t(11)=0.58$; both $p \mathrm{~s}>.05]$. There was a nonsignificant trend toward a decrease in forward displacement over the course of motion $[F(2,22)=2.94, p<.1]$.

Averaged across motion directions, there was a forward displacement after $20^{\circ}$ of motion that decreased in the course of motion, resulting in virtually no displacement after $40^{\circ}$ and a slight backward displacement after $60^{\circ}$ of motion (Figure 4B). Only the initial displacement differed from zero $\left[2.7^{\circ}, S E \pm 0.9^{\circ} ; t(11)=2.93, p<.05\right]$, whereas middle $\left[-0.2^{\circ}, S E \pm 0.7^{\circ} ; t(11)=0.32, p>.05\right]$ and final $\left[-0.4^{\circ}, S E \pm 1.2 ; t(11)=0.33, p>.05\right]$ displacements did not. An ANOVA revealed an effect of phase of motion $[F(2,22)=4.33, p<.05$; Greenhouse-Geisser corrected values]. Post hoc $t$ tests showed that the initial displacement differed from that in the middle phase of motion $(p<.05)$, but not from that in the final phase of motion.

A comparison of the results of Experiments 1A and 1B revealed strictly opposite patterns of displacement, with increasing forward displacement with motion duration for relative judgments and decreasing forward displacement for the pointing task. This was confirmed by a three-factor repeated measures ANOVA, indicating a threefold interaction $[F(2,22)=13.12, p<.001]$ between psychophysical method (relative judgment, pointing), motion direction (LR, RL), and target position (left, central, right).

\section{Discussion}

The results obtained with relative judgments (Experiment $1 \mathrm{~A}$ ) suggest that a moving sound source is localized with a forward displacement of its current position that increases in the course of the motion. This finding replicates that of Mateeff and Hohnsbein (1988, Experiment 1), who investigated the localization of an acoustic target moving in an LR direction at different points in time using relative judgments. They found a backward displacement (i.e., a shift opposite the direction of motion) of about $-4^{\circ}$ at the beginning of motion and a forward displacement of up to $12.5^{\circ}$ (with reference to stationary acoustic targets) that emerged in the middle phase of motion and increased at its end. Although the amplitudes of the displacements obtained in the present study were smaller, this overall pattern was similar to that of Mateeff and Hohnsbein.

Surprisingly, the results obtained with the pointing task (Experiment 1B) were quite different: An initial forward displacement, measured in the first phase of motion, turned into a slight backward displacement in the final phase of motion. How can these conflicting results be explained? At first, one could assume that the differences resulted from the two types of localization tasks used, which could be labeled as cognitive and sensorimotor tasks (Paillard, 1987): The relative judgments were based on a cognitive process of comparing the current positions of the acoustic target and the visual reference. On the other hand, pointing to the position of an acoustic target, which comprises motor planning and execution, may rather represent a sensorimotor measure of localization. Assuming that sensorimotor and cognitive measures are based on distinct spatial representations of auditory space (as has been demonstrated for the visual system by, e.g., Goodale \& Milner, 1992), the divergent results of pointing and relative judgment could be explained by different processing of dynamic information. Along similar lines, effects of the measuring method of localization on visual representational momentum have been explained (Kerzel, 2003b; Kerzel \& Gegenfurtner, 2003). It should be noted, however, that in these studies forward displacement of the final target position was found with pointing responses rather than with relative judgments, whereas in the present study an increase of forward displacement was found with relative judgments rather than with pointing.

The most important difference between Experiments 1A and $1 \mathrm{~B}$ might be that in the latter experiment the acoustic target moved in total darkness, whereas in Experiment 1A a visual stimulus was present during motion. A crossmodal spatial interaction of visual and acoustic stimuli could have occurred, resulting in a visual bias of sound localization (see, e.g., Lewald et al., 2001; Lewald \& Guski, 2003; Shelton \& Searle, 1980; Warren, 1970). Moreover, the visual stimulus could have cued the spatial position where the acoustic target would have been located at the moment of tactile stimulation. For example, with the visual stimulus presented at the central reference position, the subject could expect the tactile stimulus to be applied at the midpoint of the motion trajectory even before the acoustic target started. In contrast to that situation, with the pointing operation of Experiment 1B, the subjects did not have any clue about the potential target position prior to the tactile stimulation. Because these methodological factors may account for the difference in localization displacements found in Experiments 1A and 1B, a second experiment was conducted that allowed for a further comparative analysis of relative judgment and pointing.

\section{EXPERIMENT 2}

Experiment 2A was the same as Experiment 1A, with one major difference: The acoustic target was presented in total darkness, and the visual reference appeared only after the end of motion. Experiment $2 \mathrm{~B}$ comprised a pointing task in total darkness, as in Experiment 1B. We hypothesized that the contrasting patterns of displacement observed in the two parts of Experiment 1 were due to the presence of the visual reference during motion in Experiment $1 \mathrm{~A}$. If this was true, such a discrepancy should not occur between Experiments 2A and 2B.

\section{Method}

Subjects. Eleven female subjects (age range 19-39 years, mean 24.2 years) participated in this experiment. All had normal hearing and normal or corrected-to-normal vision. They were unaware of the scientific background of the study and gave their informed consent prior to inclusion in the study.

Procedure in Experiment 2A: Relative judgments. The apparatus and stimuli were as in Experiment 1A, except for the procedure in the first block, in which subjects judged the current positions of motion stimuli: Each trial started with the onset of the acoustic motion in the LR or RL direction. During motion (duration $6.8 \mathrm{sec}$ ), a tactile stimulus like the one employed in Experiment 1 was presented. With a delay of $0.6 \mathrm{sec}$ after the end of motion, the visual reference (duration $8.7 \mathrm{sec}$ ) was presented at the left $\left(-20^{\circ}\right)$, central 
$\left(0^{\circ}\right)$, or right $\left(20^{\circ}\right)$ position, which was randomly varied. The subjects estimated the target position at the moment of tactile stimulation, and the next trial started $2.1 \mathrm{sec}$ after a subject's response or-if the subject did not respond - at the end of the visual stimulus. The distances between the current target position and the visual reference were $\pm 12^{\circ}, \pm 8^{\circ}, \pm 4^{\circ}$, or $0^{\circ}$. To prevent a potential effect of lateral tactile stimulation, for each subject the position of the pressure transducer was changed between the left and right arms after half of the trials.

Procedure in Experiment 2B: Pointing. Experiment 2B was conducted after Experiment 2A, with a short break (about $20 \mathrm{~min}$ ) between the experiments. It was conducted as Experiment $1 \mathrm{~B}$ had been, except that each trial lasted for $12 \mathrm{sec}$ and 8 trials were conducted per condition (total of 72 trials; 3 target positions $\times 3$ motion conditions [LR, RL, stationary] $\times 8$ replications). These modifications were intended to improve the accuracy of sound localization.

As in Experiment 1A, audio-tactile differences in perceptual latencies were determined by a temporal order task (see above), which revealed only slight differences (mean value $+3.6 \mathrm{msec}, S E$ $\pm 5.5 \mathrm{msec}$ ). Nevertheless, the forward displacements were corrected for the shift of target position due to different perceptual latencies $\left(0.04^{\circ}, S E \pm 0.07^{\circ}\right)$.

\section{Results}

Experiment 2A: Relative judgments. Figure 5A shows the displacements obtained with relative judgments. When the target moved in the LR direction, there was a forward displacement at the left reference position and a backward displacement at the right reference position. Similarly, with RL motion, the displacement was forward at the right reference position and backward at the left and central reference positions. Only displacements in the first phase of motion differed statistically from zero [LR motion and left reference, $t(10)=2.66$, $p<.05 ; \mathrm{RL}$ motion and right reference, $t(10)=2.33, p<$ .05], whereas displacements in the middle [LR motion and central reference, $t(10)=0.07, p>.05$; RL motion and central reference, $t(10)=0.95, p>.05]$ and final [LR motion and right reference, $t(10)=0.94, p>.05$; RL motion and left reference, $t(10)=1.95, p>.05]$ phases of motion did not. The effect of position was confirmed by ANOVAs for both motion directions [LR, $F(2,20)=4.57$, $p<.05$; RL, $F(2,20)=5.41, p<.05]$.

Averaged across motion directions, there was an initial forward displacement that turned into a backward displacement in the final phase of motion (Figure 4C). However, only the initial displacement differed significantly from zero $\left[4.9^{\circ}, S E \pm 1.9^{\circ} ; t(10)=2.64, p<.05\right]$, whereas the middle displacement $\left[-0.8^{\circ}, S E \pm 1.3^{\circ}\right.$; $t(10)=0.57, p>.05]$ and the final displacement $\left[-3.4^{\circ}\right.$, $\left.S E \pm 2.2^{\circ} ; t(10)=1.55, p>.05\right]$ did not. An ANOVA indicated a main effect of phase of motion $[F(2,20)=5.63$, $p<.05$; Greenhouse-Geisser corrected value]. Also, post hoc $t$ tests showed that the initial forward displacements differed from the displacements at the middle and final phases of motion $(p<.05)$.

Experiment 2B: Pointing. As is shown in Figure 5B, LR motion induced a general forward displacement that decreased in the course of motion. Only the forward displacement at the left reference position was significantly different from zero $[t(10)=3.02, p<.05]$, whereas those at the central and right reference positions were not
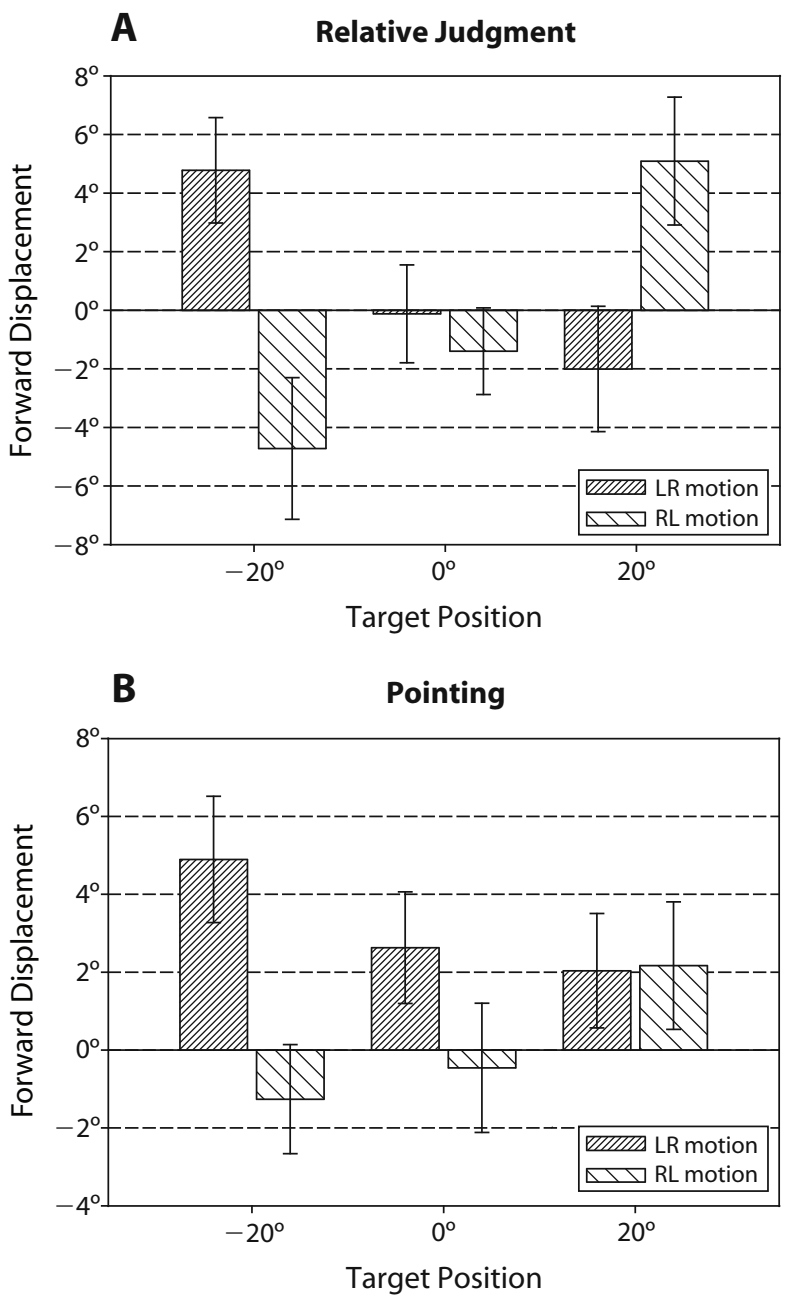

Figure 5. Mean localization displacements in Experiment 2 as a function of the target position and direction of motion, shown separately for relative judgments (A) and pointing (B). Displacements were calculated by subtracting differences obtained in the stationary conditions from those in the motion conditions. Notation conventions are as in Figure 3 , and error bars are $S E$ s across subjects $(N=11)$. $L R$, left to right; $R L$, right to left.

$[t(10)=1.83 ; t(10)=1.39 ;$ both $p \mathrm{~s}>.05]$. With RL motion, only slight displacements occurred (forward in the first phase and backward in the middle and final phases of motion) that did not differ significantly from zero [left, $t(10)=0.90$; central, $t(10)=0.28$; right, $t(10)=1.33$; all $p s>.05]$. The effect of position on forward displacement was nonsignificant with $\operatorname{LR}[F(2,20)=1.85, p>.1]$ as well as with $\operatorname{RL}[F(2,20)=2.26, p>.1]$ motion.

Averaged across motion directions, the results indicate an initial forward displacement that slightly decreased over the middle and final phases of motion (Figure 4D). Whereas the initial displacement was different from zero $\left[3.5^{\circ}, S E \pm 1.4^{\circ} ; t(10)=1.34, p<.05\right]$, those at the middle $\left[1.1^{\mathrm{o}}, S E \pm 1.1^{\mathrm{o}} ; t(10)=0.98, p>.5\right]$ and final $\left[0.4^{\mathrm{o}}, S E\right.$ $\left.\pm 1.3^{\circ} ; t(10)=0.30, p>.05\right]$ phases of motion were not. An ANOVA did not indicate an effect of phase of motion $[F(2,20)=2.73, p>.05$; Greenhouse-Geisser corrected 
value]. However, post hoc $t$ tests showed that the initial forward displacements differed from the displacement in the middle $(p<.05)$, but not from that in the final phase of motion.

A comparison of the results of Experiments $2 \mathrm{~A}$ and $2 \mathrm{~B}$ revealed that the general patterns of displacement were similar. Accordingly, a three-factor repeated measures ANOVA indicated an interaction of motion direction and target position $[F(2,20)=7.24, p<.05]$ and a main effect of target position $[F(2,20)=5.89, p<.05]$. The ANOVA did not indicate any effect of psychophysical method on displacements, nor any interaction of psychophysical method, motion direction, and target position $[F(2,20)=1.46, p>.05]$. A difference in displacements was obtained merely in the final phase of motion $[t(10)=$ 2.38, $p<.05]$ : Averaged across motion directions, there was no displacement with pointing, but a slight backward displacement with relative judgments.

\section{Discussion}

In contrast to Experiment 1, the overall patterns of displacement here did not differ between the psychophysical methods employed. With both the relative-judgment and pointing tasks, a forward displacement occurred in the first phase of motion that decreased in the further course of motion: Either it turned into a (nonsignificant) backward displacement (Experiment 2A) or it disappeared (Experiment 2B). The results of both of these experiments largely resemble those of Experiment 1B. Thus, the converse patterns of displacement observed in Experiments 1A and 1B may have primarily resulted from the presence of the visual stimulus during motion in Experiment 1A. This presence might have induced a visual bias of sound localization or acted as a frame of reference or cue to the target position that was not available in Experiments 1B and 2. Without any visual reference during motion, the position of the moving acoustic target seems to have been displaced in the direction of motion only in the initial phase of motion.

One cannot completely exclude that some effect of the retention interval occurred in Experiment 2A, in which the visual reference was always presented $600 \mathrm{msec}$ after the end of the acoustic target. Thus, the earlier the phase of the target trajectory, the more time elapsed up to the listener's response. As demonstrated by Freyd and Johnson (1987), forward displacements of the final position of a visual target declined with increasing retention interval, and even turned into a backward shift with very long retention intervals. This finding was attributed to two superimposing effects: (1) representational momentum operating immediately after perception of the inducing stimulus (causing forward displacements) and (2) "memory averaging" processes dominating after some time has elapsed. In this context, memory averaging means that all positions of the target on the motion trajectory before its ceasing are combined in memory, thus inducing an apparent shift of the remembered final position toward earlier points in time - that is, a spatial backward shift along the perceived motion trajectory. Assuming that this holds true also for acoustic targets, the pattern of displacements in Experiment 2A could be explained by assuming memory averaging of those parts of the motion trajectory that followed (or preceded) the position to be localized: With localization in the initial phase of motion, memory averaging of the subsequent trajectory may induce a forward shift; with localization in the final phase of motion, memory averaging may induce a backward shift. On the one hand, a possible interaction of the effect of retention duration and auditory representational momentum with relative-judgment tasks is thus an important issue that has to be addressed in future studies. On the other hand, it has to be emphasized that the retention interval did not play any role in the pointing task, since subjects localized the target immediately after the tactile stimulation.

\section{EXPERIMENT 3}

Results obtained with the pointing task suggest that the forward displacement of the final position of a moving acoustic target, which has been termed representational momentum in spatial hearing (Getzmann, 2005a; Getzmann et al., 2004), does not emerge until the target has ceased. To test this hypothesis, a third experiment was conducted that concentrated on the localization of the final point of motion. Employing the pointing operation used in Experiments 1B and 2B, the localization of a moving acoustic target was investigated at three different points in time: (1) shortly before the motion ceased, (2) at the moment of cessation, and (3) after cessation of the motion. On the basis of Experiments 1 and 2, it was expected that a forward displacement would occur exclusively after the cessation of the motion.

\section{Method}

Subjects. Eleven subjects with normal hearing ( 3 female, 8 male; age range 20-26 years, mean 23.6 years) participated in the experiment. All were unaware of the scientific background of the study and gave their informed consent prior to inclusion.

Procedure. The apparatus, sound stimuli, and pointing task were as in Experiments 1B and 2B. Each session consisted of three blocks. In the first block, subjects localized targets that moved along four different motion trajectories: LR motion that started at $-42^{\circ}$ and ended at either $10^{\circ}$ or $20^{\circ}$, or RL motion that started at $42^{\circ}$ and ended at either $-10^{\circ}$ or $-20^{\circ}$ (Figure 6A). The motion lasted for either $4.3 \mathrm{sec}$ (short trajectory) or $5.2 \mathrm{sec}$ (long trajectory). A tactile stimulus was presented at the moment when the target either had reached a point $20^{\circ}$ before its final position or was at that final position of motion. In the latter case, the onset of the tactile stimulus coincided with the onset of the last noise burst of the auditory (apparent) motion stimulus. The subjects were instructed to direct the hand pointer after the end of motion toward the position where the target was at the moment of tactile stimulation. Alternatively, in some trials no tactile stimulus was presented. In this condition, which was commensurate with that in our previous studies (Getzmann, 2005a; Getzmann et al., 2004), the subjects were instructed to point toward the position where the target had ceased. Each trial lasted for $12 \mathrm{sec}$. Seven LR- and seven RL-motion stimuli per condition were presented in quasirandom order. Thus, the first block comprised a total of 84 trials ( 4 motion trajectories $\times 3$ times of localization $\times 7$ replications).

In the second block, the subjects pointed to stationary target sounds (duration $4.3 \mathrm{sec}$ ) that were presented at $-20^{\circ},-10^{\circ}, 0^{\circ}, 10^{\circ}$, or $20^{\circ}$. As in Experiments $1 \mathrm{~B}$ and $2 \mathrm{~B}$, these "stationary trials" were used to correct displacements obtained with sound motion. Seven stimuli per position were presented in quasirandom order. 

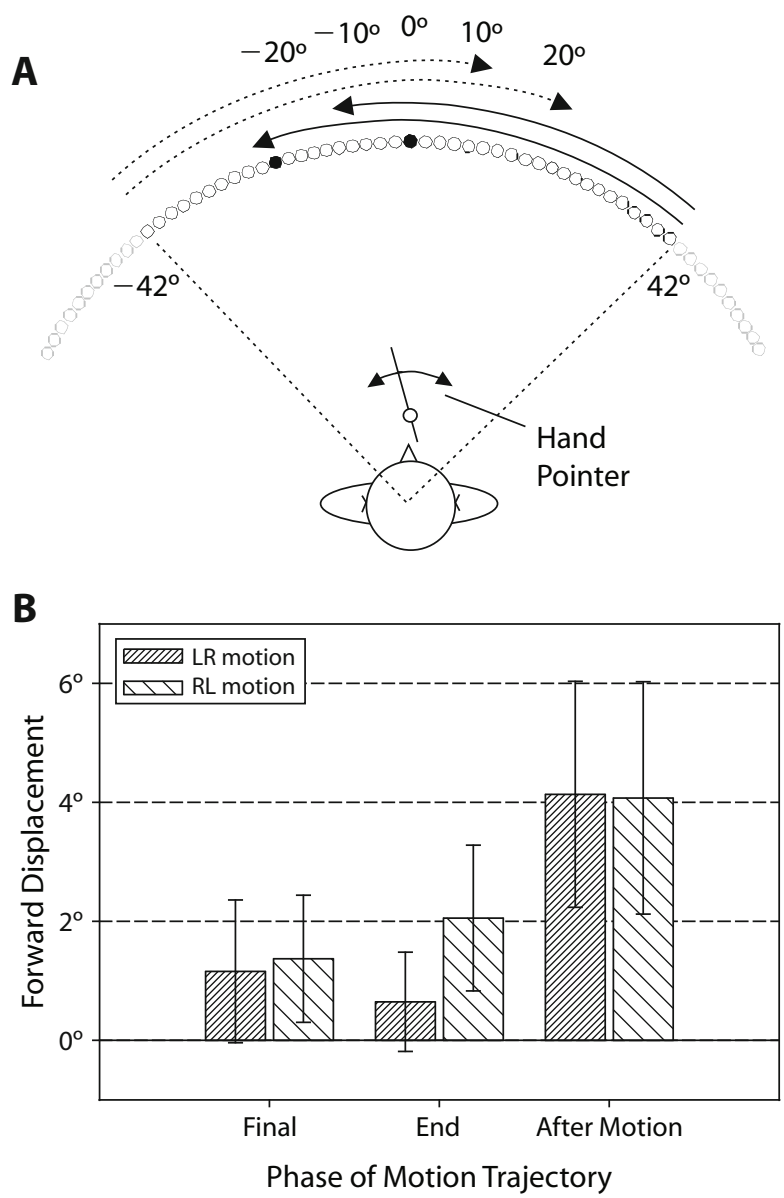

Figure 6. (A) Schematic illustration of the trajectories of leftto-right (LR, dotted arrows) and right-to-left (RL, solid arrows) motion in Experiment 3. Positive positions are to the right, and negative positions to the left, of the subject's median plane. Subjects pointed at the position where the moving acoustic target was located at the moment they received a tactile stimulus, or at the target's final position when there was no tactile stimulus. Tactile stimulation either was presented $20^{\circ}$ before the end of motion or coincided with the end of motion. The actual target positions at the moment of tactile stimulation are exemplarily marked for the RL motion that ended at $-\mathbf{2 0}^{\circ}$ (filled circles). (B) Forward displacements in Experiment 3 as a function of the phase of motion trajectory and direction of motion. The final phase refers to the moment at which the target was $20^{\circ}$ before its final position; end refers to the moment when the target reached its final position; and after motion refers to the moment after the target's disappearance. The notation conventions are as in Figure 3, and error bars are $S E$ s across subjects $(N=11)$.

In the third block, audio-tactile differences in perceptual latencies were determined through a temporal order task (see above). Again, this task revealed only slight differences (mean value $-16.4 \mathrm{msec}$, $S E \pm 29.3 \mathrm{msec}$ ), which were used to correct forward displacements for the shift of target position due to audio-tactile latency differences $\left(-0.20^{\circ}, S E \pm 0.35^{\circ}\right)$.

\section{Results}

As is shown in Figure 6B, the magnitude of the perceptual displacement depended on the phase of the motion trajectory: During the motion (i.e., $20^{\circ}$ before the final target position) and at the end of motion, the target position was slightly displaced in the direction of motion, whereas a stronger forward displacement occurred after the target's disappearance. Accordingly, a two-factor repeated measures ANOVA indicated a significant effect of phase of motion trajectory $[F(2,20)=5.28, p<.05]$, but no effect of motion direction $[F(1,10)=0.32, p>.05]$, and no interaction between the two $[F(2,20)=1.06, p>.05]$.

Averaged across motion directions, the results can be described as a slight forward displacement occurring during and at the end of motion, and a stronger forward displacement occurring after the target had disappeared (Figure 4E). Accordingly, $t$ tests indicated that only the forward displacement after the target's disappearance differed significantly from zero $\left[4.1^{\circ}, S E \pm 1.8^{\circ} ; t(10)=\right.$ 2.28, $p<.05]$, whereas displacements occurring during motion $\left[1.3^{\circ}, S E \pm 1.0^{\circ} ; t(10)=1.28, p>.05\right]$ and at the end of motion $\left[1.4^{\circ}, S E \pm 1.0^{\circ} ; t(10)=1.39, p>.05\right]$ did not. Additional post hoc $t$ tests indicated that the forward displacement after the target's disappearance was significantly $(p<.05)$ larger than displacements during and at the end of motion.

\section{Discussion}

There was a slight (statistically nonsignificant) forward displacement of the target position during the motion, quite similar to that observed in Experiment 2B in the middle and final phases of motion (cf. Figure 4D). At the end of motion, a nonsignificant tendency toward displacement occurred that did not differ from that during the motion. However, without a tactile stimulus (when determining the moment of target localization), the final position appeared to be shifted in the direction of motion. This result corresponds with the previously reported shift of the final position of a moving sound, and thus replicates the effect of representational momentum in spatial hearing (Getzmann, 2005a; Getzmann et al., 2004). The magnitude of forward displacement of the final position $\left(4.1^{\circ}\right)$ was quite similar to that found in our preceding studies (about $3.4^{\circ}$ and $3.5^{\circ}$ ). Taken together, the results indicate that the localization of a moving acoustic target was quite precise as long as the target was present. After its disappearance, the final position appeared to be shifted in the direction of motion.

One might argue that the presence of a tactile stimulus, as used here, could counteract the perception of forward displacements. There is evidence that the presentation of a distractor at the moment a moving target vanishes can affect forward displacements in visual representational momentum. In particular, judgments of the final position were biased toward the location of the distractor (Kerzel, 2002). It was suggested in that study that the position of the distractor interfered with the remembered final target position. Also, backward displacements of final target positions were reported when a task-relevant stationary stimulus was presented at the moment the target vanished (Müsseler et al., 2002). It should be noted, however, that in these studies both target and distractor were visual objects. At present, it is unclear whether or not spatiotemporal interactions of target and distractor positions occur when an acoustic target is combined with a tactile distractor. 
It is also conceivable that the presence of the tactile stimulus reduced the forward displacement by distracting attention from the moving acoustic target. In the visual domain, numerous studies have demonstrated that allocation of attention can influence displacement effects (Hayes \& Freyd, 2002; Joordens et al., 2004; Kerzel, 2003a; Munger \& Owens, 2004; for a review, see Hubbard, 2005). However, most of those studies indicated more pronounced forward displacements when distractors were present. ${ }^{2}$ Thus, the possibility seems unlikely that the absence of a displacement effect at the moment of the target's cessation in this experiment was the result of an allocation of attention by the tactile stimulus.

The pattern of displacement effects obtained here resembles that reported by Joordens et al. (2004). In a clockwatching task, they failed to find any momentum-like bias during continuous motion of a visual target (Experiment 1 and single-task condition of Experiment 2); after the target's cessation, however, a forward displacement of the final position occurred (Experiment 3). It was proposed that a compensation process, counteracting the effect of representational momentum, reduced (and even eliminated) forward displacements. This compensation may result from controlled processes initiated by the observers, which may depend on the experimental context. The tendency to compensate might be reduced when the target does not continue to move, leaving a strong momentum effect that, in turn, causes forward displacement (Joordens et al., 2004).

\section{GENERAL DISCUSSION}

The present study investigated the localization of a moving acoustic target at different points of horizontal motion trajectories, employing two psychophysical methods: relative judgment and pointing. Although subjects were generally able to localize the sound stimuli with both methods, there was a specific pattern of perceptual displacement: Without any visual reference in total darkness, the pattern could be best described as an initial forward displacement that decreased in the further course of motion. With relative judgments, this displacement turned into a backward shift in the final phase of motion (Experiment 2A), whereas with pointing slight displacements remained in the middle and final phases of motion (Experiments 1B and 2B) and at the final target position (Experiment 3). However, after disappearance of the target, its final position was significantly displaced in the direction of motion (Experiment 3 ).

In Experiment 1A, an increasing forward displacement occurred in the course of motion, similar to the displacement reported by Mateeff and Hohnsbein (1988). The seeming conflict of this result and those of Experiments $1 \mathrm{~B}$ and 2 can be attributed to divergent experimental settings - namely, the visual reference presented during the auditory motion in Experiment 1A. Experiment 2A ruled out the possibility that the forward displacement in the final phase of motion in Experiment 1A was related to the shift of the final position after the target's disappearance, because without a preceding reference stimulus there was a backward, rather than a forward, shift that increased in the course of motion.

Interestingly, a strong forward displacement occurred in the initial phase of motion, irrespective of the localization method. At that point in time, the tactile stimulus was presented when the auditory target had covered only a relatively small angle. The forward displacement is analogous to that observed in the Fröhlich effect, in which subjects determine the onset position of an object in motion (see, e.g., Baldo \& Klein, 1995; Müsseler \& Aschersleben, 1998; Müsseler et al., 2002). As in the Fröhlich effect, forward displacements in the initial phase of motion could be based on attentional processes: Because the target started randomly in the left or right hemisphere, subjects did not know the starting point in advance. The initial phase of the trajectory may therefore have received less attention, because it takes some time before spatial attention is focused on the current target position. Given that attention is a necessary prerequisite for conscious processing of spatial information, the representation of the initial phase of the trajectory could be insufficient, so that the target appears to be shifted toward later positions - that is, in the direction of motion.

This attention-based explanation for the initial forward displacement is compatible with findings indicating that auditory attention oriented to a specific location in space enhances the precision of auditory localization (e.g., Sach, Hill, \& Bailey, 2000; Spence \& Driver, 1994; for a review, see Scharf, 1998). Also, the results of Experiment 1A can support such an attention-based account: When the visual reference stimulus was visible before the target started, no forward displacement occurred. In this case, the reference stimulus could initiate a "preliminary" shift of spatial attention toward the target position that reduced the time needed for the shift of attentional focus. Thus, the representation of the initial phase of the motion trajectory was strengthened. Similarly, a preceding cue that indicated the onset position of a subsequent motion has been shown to reduce the forward displacement in the Fröhlich effect in both the visual (Müsseler \& Aschersleben, 1998, Experiment 4) and auditory (Getzmann, 2005b) modalities.

How are the present findings related to the representational momentum in spatial hearing? At first, these results clearly contradict our initial hypothesis, derived from the results of Mateeff and Hohnsbein (1988), which predicted an increase of forward displacement during motion. Unlike this hypothesis, the forward displacements obtained here during motion were relatively small, as compared with that of the final target position after the target's disappearance. Also, displacements decreased up to the final point, where they were almost absent, or even opposite the direction of motion. So far, the theoretical background of representational momentum has referred mainly to visual objects in motion (see, however, the discussion of representational momentum in pitch perception by Freyd, Kelly, \& DeKay, 1990), but several assumptions could be readily adapted to account for the present findings in auditory motion perception. Functionally, the perception of motion is of vital importance in everyday life. In particular, faced with the problem of planning or avoiding 
contact with an object in motion, the perceptual system must represent motion. In order to compensate for delays within the neural pathway, the perceptual system should incorporate future positions of the moving object. In order to catch an object in motion, for example, the perceptual system must compensate for the object's movement during the period from initial detection of the object to the completion of processing of its spatial position and the initiation of a motor response. Representational momentum might serve to adjust the object's representation to the position where the object would be in the very near future, thus "bridging the gap between perception and action" (Hubbard, 2005, 2006).

It has been suggested that within the visual system, this compensation is at least in part based on mental extrapolation of past trajectory information (Nijhawan, 1994). However, psychophysical results have suggested that compensation does not occur at early stages of visual processing, but at a stage at which retinotopic information is transformed into egocentric space (Kerzel \& Gegenfurtner, 2003). Thus, extrapolation in the motor (rather than visual) system may overcome processing delays inherent in the visual system. Assuming that the spatial representation of a moving acoustic target also lags behind the actual position, mental extrapolation of dynamic auditory information could subserve the localization of a moving sound source as well. Supporting this reasoning, displacements observed with the pointing method were generally small during motion, and in particular, the perceived target positions did not lag behind the actual positions. The effect of mental extrapolation should occur when the moving target abruptly disappears. In this case, which was unpredictable for the perceptual system, the dynamic representation did not stop immediately but overshot the final target position in the direction of motion.

Mental extrapolation does not require that the moving object be associated with physical momentum: It has been proposed that momentum is a general property of representational systems in which time is a fully integrated dimension, and that momentum can be found for any type of continuous change (Freyd et al., 1990). A causal relation between physical and representational momentum does not appear to be necessary; instead, the perceptual system could use the rate of change of a continuously changing event as an intrinsic component for automatically extrapolating future states of the event. Regarding representational-momentumlike phenomena in dimensions that cannot be directly associated with physical-momentum-like phenomena (e.g., tone pitch, Freyd et al., 1990; or brightness, Brehaut \& Tipper, 1996), this argumentation appears plausible.

Interestingly, with the relative-judgment method, a backward displacement of target position emerged during motion (Experiment 2A). In particular, in the final phase of motion, there was a significant difference between the relative-judgment and pointing task results: Although manual pointing was quite precise, the relative judgments obviously lagged behind the actual target position. Thus, mental extrapolation of dynamic information may play a major role in sensorimotor operations - that is, in operations that comprise motor planning and execution, as in the present pointing task. This is in line with findings on visual representational momentum that have indicated that stronger forward displacements after the target's cessation occur with motor responses (e.g., manual pointing) than with relative judgments (Kerzel, 2003b; Kerzel \& Gegenfurtner, 2003). Assuming that motor actions and cognitive judgments are based on distinct representations (Goodale \& Milner, 1992), our data thus may provide further support for the view that the motor system anticipates future positions to a larger degree than does the cognitive system, in order to compensate for neuronal delays and to ensure precise goal-directed movements.

\section{AUTHOR NOTE}

The authors thank J. Hohnsbein, T. L. Hubbard, S. Mateeff, and an anonymous reviewer for valuable comments on previous versions of the manuscript, and P. Dillmann for preparing software and electronic equipment. Correspondence concerning this article should be addressed to S. Getzmann, Ruhr-Universität Bochum, Fakultät für Psychologie, Kognitions- und Umweltpsychologie, Universitätsstr. 150, D-44780 Bochum, Germany (e-mail: stephan.getzmann@ruhr-uni-bochum.de).

\section{REFERENCES}

Baldo, M. V. C., \& Klein, S. A. (1995). Extrapolation or attention shift? Nature, 378, 565-566.

Brehaut, J. C., \& Tipper, S. P. (1996). Representational momentum and memory for luminance. Journal of Experimental Psychology: Human Perception \& Performance, 22, 480-501.

Cox, D. R. (1970). The analysis of binary data. London: Methuen.

Dong, C.-J., Swindale, N. V., Zakarauskas, P., Hayward, V., \& CYNADER, M. S. (2000). The auditory motion aftereffect: Its tuning and specificity in the spatial and frequency domains. Perception \& Psychophysics, 62, 1099-1111.

Freyd, J. J. (1987). Dynamic mental representations. Psychological Review, 94, 427-438.

FrEYD, J. J., \& FinKE, R. A. (1984). Representational momentum. Journal of Experimental Psychology: Learning, Memory, \& Cognition, 10, 126-132.

Freyd, J. J., \& Johnson, J. Q. (1987). Probing the time course of representational momentum. Journal of Experimental Psychology: Learning, Memory, \& Cognition, 13, 259-268.

Freyd, J. J., Kelly, M. H., \& DeKay, M. L. (1990). Representational momentum in memory for pitch. Journal of Experimental Psychology: Learning, Memory, \& Cognition, 16, 1107-1117.

FröHLICH, F. W. (1923). Über die Messung der Empfindungszeit [Measuring the time of sensation]. Zeitschrift für Sinnesphysiologie, 54, 58-78.

Getzmann, S. (2005a). Representational momentum in spatial hearing does not depend on eye movements. Experimental Brain Research, 165, 229-238.

Getzmann, S. (2005b). Shifting the onset of a moving sound source: A Fröhlich effect in spatial hearing. Hearing Research, 210, 104-111.

Getzmann, S., Lewald, J., \& Guski, R. (2004). Representational momentum in spatial hearing. Perception, 33, 591-599.

Gilkey, R. H., \& Anderson, T. R. (1997). Binaural and spatial hearing in real and virtual environments. Mahwah, NJ: Erlbaum.

Goodale, M. A., \& Milner, A. D. (1992). Separate visual pathways for perception and action. Trends in Neurosciences, 15, 20-25.

Grantham, D. W. (1992). Adaptation to auditory motion in the horizontal plane: Effect of prior exposure to motion on motion detectability. Perception \& Psychophysics, 52, 144-150.

Hayes, A. E., \& Freyd, J. J. (2002). Representational momentum when attention is divided. Visual Cognition, 9, 8-27.

Hubbard, T. L. (2005). Representational momentum and related displacements in spatial memory: A review of the findings. Psychonomic Bulletin \& Review, 12, 822-851.

HubBard, T. L. (2006). Bridging the gap: Possible roles and contributions of representational momentum. Psicológica, 27, 1-34.

Joordens, S., SpaleK, T. M., Razmy, S., \& VAN Duijn, M. (2004). A 
Clockwork Orange: Compensation opposing momentum in memory for location. Memory \& Cognition, 32, 39-50.

KerzeL, D. (2002). Attention shifts and memory averaging. Quarterly Journal of Experimental Psychology, 55A, 425-443.

KerzeL, D. (2003a). Attention maintains mental extrapolation of target position: Irrelevant distractors eliminate forward displacement after implied motion. Cognition, 88, 109-131.

KERZEL, D. (2003b). Mental extrapolation of target position is strongest with weak motion signals and motor responses. Vision Research, $\mathbf{4 3}$, 2623-2635.

Kerzel, D. (2005). Representational momentum beyond internalized physics: Embodied mechanisms of anticipation cause errors in visual short-term memory. Current Directions in Psychological Science, 14, 180-184.

Kerzel, D. (2006). Why eye movements and perceptual factors have to be controlled in studies on "representational momentum." Psychonomic Bulletin \& Review, 13, 166-173.

Kerzel, D., \& Gegenfurtner, K. R. (2003). Neuronal processing delays are compensated in the sensorimotor branch of the visual system. Current Biology, 13, 1975-1978.

KInG, A. J., \& PAlmer, A. R. (1985). Integration of visual and auditory information in bimodal neurones in the guinea-pig superior colliculus. Experimental Brain Research, 60, 492-500.

Lewald, J., Dörrscheidt, G. J., \& Ehrenstein, W. H. (2000). Sound localization with eccentric head position. Behavioural Brain Research, 108, 105-125.

Lewald, J., Ehrenstein, W. H., \& Guski, R. (2001). Spatio-temporal constraints for auditory-visual integration. Behavioural Brain Research, 121, 69-79.

LEWALD, J., \& GUSKI, R. (2003). Cross-modal perceptual integration of spatially and temporally disparate auditory and visual stimuli. Cognitive Brain Research, 16, 468-478 .

LUTFI, R. A., \& WANG, W. (1999). Correlational analysis of acoustic cues for the discrimination of auditory motion. Journal of the Acoustical Society of America, 106, 919-928.

Malone, B. J., Scott, B. H., \& Semple, M. N. (2002). Contextdependent adaptive coding of interaural phase disparity in the auditory cortex of awake macaques. Journal of Neuroscience, 22, 4625-4638.

Mateeff, S., \& Hohnsbein, J. (1988). Dynamic auditory localization: Perceived position of a moving sound source. Acta Physiologica et Pharmacologica Bulgarica, 14, 32-38.

Munger, M. P., \& Owens, T. R. (2004). Representational momentum and the flash-lag effect. Visual Cognition, 11, 81-103.

Müsseler, J., \& Aschersleben, G. (1998). Localizing the first position of a moving stimulus: The Fröhlich effect and an attention-shifting explanation. Perception \& Psychophysics, 60, 683-695.

Müsseler, J., Stork, S., \& Kerzel, D. (2002). Comparing mislocalizations with moving stimuli: The Fröhlich effect, the flash-lag, and representational momentum. Visual Cognition, 9, 120-138.

NeELon, M. F., \& Jenison, R. L. (2003). The effect of trajectory on the auditory motion aftereffect. Hearing Research, 180, 57-66.

NiJhawan, R. (1994). Motion extrapolation in catching. Nature, 370, 256-257.

PAILlaRd, J. (1987). Cognitive versus sensorimotor encoding of spatial information. In P. Ellen \& C. Thinus-Blanc (Eds.), Cognitive pro- cesses and spatial orientation in animal and man (Vol. 2, pp. 43-77) Dordrecht: Martinus Nijhoff.

Perrott, D. R., Manligas, C. L., \& Pacheco, S. (1988). Minimum audible movement angle thresholds for broadband noise: Effects of the initial location of the source. Journal of the Acoustical Society of America, 84(Suppl. 1), S80-S81.

Perrott, D. R., \& Musicant, A. D. (1977). Minimum auditory movement angle: Binaural localization of moving sound sources. Journal of the Acoustical Society of America, 62, 1463-1466.

SACH, A. J., Hill, N. I., \& BAIley, P. J. (2000). Auditory spatial attention using interaural time differences. Journal of Experimental Psychology: Human Perception \& Performance, 26, 717-729.

Scharf, B. (1998). Auditory attention: The psychoacoustical approach. In H. Pashler (Ed.), Attention (pp. 75-117). Hove, U.K.: Psychology Press.

Shelton, B. R., \& Searle, C. L. (1980). The influence of vision on the absolute identification of sound-source position. Perception $\&$ Psychophysics, 28, 589-596.

Spence, C. J., \& Driver, J. (1994). Covert spatial orienting in audition: Exogenous and endogenous mechanisms. Journal of Experimental Psychology: Human Perception \& Performance, 20, 555-574.

Strybel, T. Z., Manligas, C. L., \& Perrott, D. R. (1992). Minimum audible movement angle as a function of the azimuth and elevation of the source. Human Factors, 34, 267-275.

Thornton, I. M., \& HubBard, T. L. (2002). Representational momentum: New findings, new directions. Visual Cognition, 9, 1-7.

WARREN, D. H. (1970). Intermodality interactions in spatial localization. Cognitive Psychology, 1, 114-133.

Wilson, W. W., \& O’NeILl, W. E. (1998). Auditory motion induces directionally dependent receptive field shifts in inferior colliculus neurons. Journal of Neurophysiology, 79, 2040-2062.

\section{NOTES}

1. One might argue that subjects were aware of the fact that each single noise burst was emitted from one loudspeaker (i.e., the impression of motion resulted from a succession of stationary sound sources). The movement angle of each sound stimulus was, however, as small as $0.6^{\circ}\left(=12^{\circ} / \mathrm{sec} * 50 \mathrm{msec}\right)$, which is clearly below the minimum audible movement angle (MAMA; i.e., the smallest angular distance a moving sound must traverse to be just discriminable from a stationary source) Perrott, Manligas, and Pacheco (1988), for example, reported that the MAMA for broadband sounds moving at a velocity of $20 \% \mathrm{sec}$ is in the range of $1.1^{\circ}$ (when the source was at $0^{\circ}$ azimuth) and $3.5^{\circ}$ (at a more lateral position). Thus, it is unlikely that subjects perceived the "stepwise" displacement of the sound source.

2. In the study of Kerzel (2003a), distracting irrelevant stimuli eliminated forward displacements, and even caused backward displacements of the final target position. However, in contrast to the other studies we have mentioned, distractors were presented after the target had vanished - that is, during the retention interval. This could possibly explain the divergent result (Hubbard, 2005).

(Manuscript received November 28, 2005; revision accepted for publication January 13, 2007.) 